\title{
The barrel cortex as a model to study dynamic neuroglial interaction
}

Christian Giaume $^{1 *}$, Miguel Maravall ${ }^{2}$, Egbert Welker $^{3}$ and Gilles Bonvento ${ }^{4}$

${ }^{1}$ Inserm U840, Collège de France, Paris, France

${ }^{2}$ Instituto de Neurociencias de Alicante UMH-CSIC, 03550 Sant Joan d'Alacant, Spain

${ }^{3}$ DBCM, Université de Lausanne, Lausanne, Switzerland

${ }^{4}$ CEA, Orsay, France

* Correspondance:

Dr Christian Giaume

INSERM U840, Collège de France

11 place Marcelin Berthelot

75005 Paris, France

Tel: 33144271222

Fax: 33144271268

Email: christian.giaume@college-de-france.fr

Short title: Neuroglial interaction in the barrel cortex

Key words: somatosensory cortex, astrocytes, glial cells

Abstract words account: 146

Text words account: 5966

Number of figures: 9 


\begin{abstract}
:
There is increasing evidence that glial cells, in particular astrocytes, interact dynamically with neurons. The well-known anatomo-functional organization of neurons in the barrel cortex offers a suitable and promising model to study such neuroglial interaction. This review summarizes and discusses recent in vitro as well as in vivo work demonstrating that astrocytes receive, integrate and respond to neuronal signals. In addition, they are active elements of brain metabolism and exhibit a certain degree of plasticity that affects neuronal activity. Altogether these findings indicate that the barrel cortex presents glial compartments overlapping and interacting with neuronal compartments and that these properties help define barrels as functional and independent units. Finally, this review outlines how the use of the barrel cortex as a model might in the future help to address important questions related to dynamic neuroglia interaction.
\end{abstract}




\section{1/ Introduction}

During the last decade our understanding of the contribution of glial cells to the integration and processing of neuronal information has advanced significantly mostly thanks to the discovery of active interactions between astrocytes and neurons. This dialog has now been demonstrated under physiological conditions for in vitro as well as in vivo models in which astrocytes were shown contributing to modulate neuronal excitability, activity and plasticity (Haydon and Carmignoto 2006; Verkhratsky and Toescu 2006; Volterra and Meldolesi 2005). These findings have even generated new and provocative concepts such as those of "tripartite synapse" and "gliotransmission" (Araque and others 1999; Volterra A 2002). Moreover, this interaction seems to play a role in several brain pathologies or psychiatric disorders, such as epilepsy and schizophrenia (Giaume and others 2007; Halassa and others 2007). However, to make further progress in this emerging field of neuroscience there is a clear need for models in which neuronal organization and physiology exhibit particular properties. An ideal model would be a brain area in which neuronal anatomy and circuitry are widely documented, which is easily accessible for in vitro (acute brain slices) as well as for non-invasive in vivo (two-photon microscopy) approaches and where functional units can be specifically activated or silenced. One model that fulfils these requirements is the region of the rodent somatosensory cortex that contains the whiskers' representation. In layer IV of this cortical area neurons are arranged in discrete clusters, called "barrels", which receive topographically organized inputs from their respective principal whiskers on the contralateral side of the face (Woolsey and Van der Loos 1970). Indeed, in a given barrel the majority of neurons preferentially respond to the stimulation of a single whisker, although they also integrate information from adjacent whiskers (ArmstrongJames and Fox 1987). Each barrel is an element of a functional column that spreads from layer II/III to layer VI (Petersen and Sakmann 2001).

Whiskers allow rodents to discriminate different roughnesses and texture identities (Carvell and Simons 1990; Guic-Robles and others 1989), to detect the width of an aperture (Krupa and others 2001), to locate objects (Knutsen and others 2006; Mehta and others 2007) and to evaluate gap-width (Hutson and Masterton 1986). This 
information is collected mainly by active sensing, i.e. by whisker motion that is actively controlled, often with fine resolution in space and time (Kleinfeld and others 2006). The barrel cortex mediates whisker-based tactile perception: ablating the barrel cortex can destroy an animal's ability to carry out a tactile location task (Guic-Robles and others 1989; Hutson and Masterton 1986).

The present review provides a small introduction to the neuronal organization of the barrel cortex and summarizes what is already known about the properties of astrocytes in the barrel cortex and how these glial cells interact actively with their neighboring neurons. However, many aspects of this interaction remain to be resolved: thus, we also outline how the barrel cortex model can help address key questions regarding dynamic neuroglia interactions.

\section{2/ Micro-organization of neuronal circuits in the barrel cortex}

Counting cell numbers suggests that, in addition to somatotopy, additional principles must govern the organization of barrel columns: as in other sensory systems, there is considerable magnification in the number of cortical neurons per receptor axon, with most of the roughly 50-fold increase (Lee and Woolsey 1975; Welker and Van der Loos 1986) occurring in the step from thalamic nucleus to cortex (Land and others 1995). This enormous increase allows an exquisitely intricate organization of neuronal circuits, which is likely to underlie sensory-motor integration. Several excellent reviews have recently focused on the organization and function of the barrel cortex and whisker system (Alloway 2008; Brecht 2007; Diamond and others 2008; Petersen 2007). Here we concentrate on specific aspects of barrel cortex organization where the importance of neuroglial interaction may be best observed.

\section{Loops and parallel pathways for active sensing}

In common with other tactile pathways, the whisker-to-barrel system has a simple feedforward structure, with cortical processing stages located just three synapses away from the sensory receptors. A great deal of the system's complexity is due to the existence of feedback loops and parallel pathways: these loops serve sensory-motor interaction 
necessary for active whisking exploration. A major stream is the "classical" lemniscal pathway that arises at the principal trigeminal nucleus and runs through the dorsomedial section of the ventral posterior medial (VPM) thalamic nucleus before innervating individual barrels in layer IV and providing weaker inputs to deep layer $\mathrm{V}$, layer $\mathrm{VI}$ and layer III. This is thought to be the main pathway involved in encoding of sensory signals related to texture and shape discrimination (Alloway 2008; Diamond and others 2008). However, at least three other pathways are known to exist: the paralemniscal pathway that runs through the rostral part of the interpolaris trigeminal nucleus and posterior medial thalamic nucleus (Diamond and others 1992), the extralemniscal pathway through the caudal part of the interpolaris nucleus and ventrolateral section of the VPM nucleus (Pierret and others 2000; Yu and others 2006), and a recently discovered pathway that projects from the principal trigeminal nucleus and runs through a strip of the VPM and through the zona incerta, and mediates the merging of sensory and motor signals (Urbain and Deschenes 2007).

Intricate cortical microcircuits implement precise timing and interdigitated processing of whisking and touch

Does the segregation into separate pathways persist in the cortex? Circuits do remain separated within the barrel cortex, at least to some degree: in layer IV, barrels are the cortical targets of the lemniscal pathway, while the other pathways pass through interbarrel regions with lower cell densities - i.e. the so-called "septa"; projections from the other pathways reach other layers as well (Bureau and others 2006; Kim and Ebner 1999; Shepherd and Svoboda 2005).

The lemniscal pathway enters the cortex via thalamocortical synapses onto layer IV neurons (Agmon and Connors 1991; Simons 1978). This projection forms the strongest, most efficacious whisker input to the cortical circuit (Bruno and Sakmann 2006; Gil and others 1999). Substantial disynaptic inhibition is evoked by the same thalamocortical afferents that provide excitation (Gabernet and others 2005; Gil and Amitai 1996; Porter and others 2001; Swadlow and Gusev 2000). This endows the layer IV postsynaptic response to a whisker stimulus with a characteristic pattern of excitation followed by 
inhibition: the resulting short time window for spike generation (Gabernet and others 2005; Wilent and Contreras 2005) gives rise to brief, transient, temporally precise spiking responses (Simons 1978; Simons and Carvell 1989). Consequently, barrel cortex responses to whisker stimulation carry substantial stimulus information in their timing, and their high precision is important for object localization and texture encoding (Arabzadeh and others 2006; Arabzadeh and others 2005).

The cortical circuits underlying these strong and precise responses mature within a short time window in the first postnatal week, during which fast postsynaptic excitation and powerful postsynaptic inhibition emerge. Activation of layer IV neurons switches from an "integrating" response mode with slow depolarizations and large receptive fields, to the precise response mode characterized by larger, sharper postsynaptic responses (Daw and others 2007). Note that these changes occur a few days before the onset of whisking, coincide with the restriction of the metabolic activation to single barrels (Melzer et al., 1985) and happen in conjunction with large developmental changes at P5-P10 in glutamate transporter expression in astrocytes (see chapter 5).

Excitatory neurons in barrels have dendritic arbors that sample the home barrel densely but do not explore layer IV beyond the limits of the barrel (Feldmeyer and others 1999; Petersen and Sakmann 2001; Schubert and others 2003). The high density of neurons and connections, interrupted at barrel borders, is mirrored by gap junction-mediated networks of astrocytes that form glial compartments (see chapter 4). Beyond layer IV, vertical projections to supragranular and infragranular layers make up a "canonical" barrel-related excitatory circuit (Lubke and Feldmeyer 2007). As illustrated in figure 1, horizontal integration between these barrel microcircuits occurs mainly at the level of layers II/III. Information flows from layer IV through to layers II/III and then to infragranular neurons where the output of the lemniscal pathway is generated. Astrocytes respond to activation of layer IV but not layers II/III, consistent with a systematic position in the overall flow of activity (see chapter 5 ). Meanwhile, thalamic inputs associated with the extralemniscal and paralemniscal pathways are not processed within barrels, but are instead processed by neurons located outside the 
barrels (see above); finally, lemniscal and paralemniscal information converge in layer Va (Feldmeyer and others 2005; Manns and others 2004; Schubert and others 2006). Accordingly, an important issue concerns how astrocytes are distributed relative to these pathways (see chapter 3 ).

\section{3/ Spatial distribution of astrocytes in the barrel cortex}

Considering the spatial organization of layer IV neurons into individual barrels, an intriguing issue arose how astrocytes are organized relative to neuronal pathways. Furthermore, is the spatial organization of astrocytes different between barrels and septa? Immunohistological studies indicate that the proportion of astrocytes versus neurons is about 0.8 based on $\mathrm{S} 100$ and NeuN stainings, respectively (Irintchev and others 2005). During barrel development, the analysis of astrocyte density has reported that GFAP-positive cells are radially oriented with a preferential location along the septa, thus sharply delineating individual barrels. In more mature mice GFAP-positive cells are present throughout the barrel field and are no longer confined to barrel sides (Cooper and Steindler 1986). Similar observation of a uniform distribution of astrocytes was also reported for 10 to 20 day-old mice based on sulforhodamine 101 or S100 stainings (Houades and others 2008; Schipke and others 2008). Using sulforhodamine B and biocytin injection of astrocytes it was shown that the size and shape of individual astrocytes are the same between those inside the barrel and those in the septum (Houades and others 2008) - invariably of the protoplasmic type. These observations point to a homogenous distribution of astrocytes characterized by a morphological pattern that is independent of the barrel compartment in which they occur. This morphological differentiation is accomplished around postnatal day 10. However, despite the homogeneous morphology, the expression of some functional astrocyte-specific markers, such as the glutamate transporters GLAST and GLT-1 and the glutamine synthetase, all highly expressed early during cortical development, is confined to the barrel hollows. In tangential sections their expression demonstrate barrel-like patterns at least until the end of the second week post-natal (Voutsinos-Porche, 2003b; (Takasaki and others 2008). So, despite a rather uniform morphology and spatial distribution, different astrocyte sub-populations (in terms of expression of specific markers and 
specific function) could cohabit in layer IV of the barrel cortex, as neurons do (see also chapter 4). These sub-populations could modulate in a distinct manner the neuronal activity in the leminiscal pathway (within the hollow of the barrel) and the pathways passing through the septal regions.

\section{4/ Network organization of astrocytes in the barrel cortex}

In the central nervous system, neurons communicate via electrical synapses comprising gap junction channels, as well as via the more widespread chemical synapses. Gap junctions occur throughout the mammalian brain and are made of proteins called connexins (Cxs) that allow ionic currents and small organic molecules to pass directly between cells. In the barrel cortex, neurons were shown to express the neuronal specific Cx, Cx36, at a significantly higher level compared to most other regions (Liu and Jones 2003), consistent with the electrical transmission reported to occur between GABAergic inhibitory interneurons (Galarreta and Hestrin 1999; Gibson and others 1999). However, although Cx expression was first studied between neurons at electrical synapses, glial cells, and in particular astrocytes are the cell population that expresses the highest amount of Cxs in the brain (Giaume and McCarthy 1996).

In astrocytes, two main Cxs prevail, Cx43 and Cx30 (Nagy and Rash 2000) which underlie a network organization of communicating cells (Houades and others 2006). Interestingly, in the somatosensory cortex their expression is up-regulated within the barrels compared to septa and other cortical layers (Houades and others 2008). In this study, the functional status of gap junctions was also tested in astrocytes by patchclamp injections of biocytin and sulforhodamine B. Results indicated that gap junctionmediated communication (indexed by biocytin intercellular diffusion) is restricted in the barrel-to-barrel direction (Fig. 2A). In addition, the enrichment of $\mathrm{Cx}$ expression and the transversal restriction of the gap junction-mediated dye-coupling are not observed in a transgenic mouse (MAOA knock-out) lacking the barrel organization (Cases and others 1995) while both are restored in a double knock-out (MAOA/5HT1b knock-out) characterized by a normal barrel field (Rebsam and others 2002). Analysis of sulforhodamine B dye coupling properties indicated that astrocytes located between two 
barrels are either weakly or not coupled whereas its diffusion within a barrel is favored towards its center (Fig. 2B) (Houades and others 2008). Taken together, these observations indicate that the occurrence of gap junctional communication confined within a barrel and oriented toward its center results from combination of subpopulations of astrocytes with different Cxs expression levels. The functional consequence is the restriction of glial communication within each barrel comparable to the organization of the excitatory neuronal circuits (chapter 2).

\section{5/ Neuroglial interaction and calcium signaling properties of astrocytes}

Although astrocytes are defined as non-excitable cells, they play an active role in information processing by establishing dynamic interaction with neurons (Haydon and Carmignoto 2006; Volterra and Meldolesi 2005). The calcium signaling properties of astrocytes confer a form of excitability and represent the molecular basis for their dialog with neighboring neurons. To achieve this task astrocytes have developed a phenotype that allows them not only to receive and respond to neuronal signal, but also to integrate and propagate neuronal signals.

Much evidence indicates that astrocytes, studied in ex vivo as well as in vivo preparations, express membrane receptors for most neurotransmitters and endogenous compounds (peptides, cytokines, hormones, bioactive lipids ...) allowing them to sense neuronal signals (Kettenmann and Steinhauser 2005). While it is argued that astrocytes "talk to" neurons by releasing "gliotransmitters", the cellular mechanism by which this communication occurs is still under debate (Agulhon and others 2008). There are reports of calcium-dependent release of glutamate, ATP and D-serine by astrocytes, and several mechanisms have been proposed to be involved in this process (vesicules, Cx hemichannels, lysosomes, ionotropic purinergic receptors...) (Deitmer and others 2006). When a signal received by an astrocyte leads to an increase in intracellular calcium concentration $\left(\left[\mathrm{Ca}^{2+}\right]_{i}\right)$, the information contained in the signal can be encoded temporally, by monophasic or oscillatory responses, and spatially through the generation of intracellular and intercellular calcium waves (Cotrina and Nedergaard 2002). Interestingly, intercellular astrocytic calcium waves (that spread at a velocity of about 15 
$\mu \mathrm{m} / \mathrm{s}$ ) have been proposed to provide "an extraneuronal pathway for rapid long-range signal transmission within the CNS" (Cornell-Bell and others 1990). While they can trigger neuronal responses in vitro (Dani and others 1992), their occurrence and their extent in vivo is still under debate (Agulhon and others 2008; Fiacco and McCarthy 2006; Scemes and Giaume 2006).

The barrel cortex is a well-adapted model to study calcium signaling in astrocytes and its incidence on neuronal activity. Indeed, an exemplification of the practical advantages of barrel cortex for in vivo imaging was the observation of spontaneous coordinated $\left[\mathrm{Ca}^{2+}\right]_{1}$ events in single astrocytes imaged within barrel columns of the anesthetized rat (Hirase and others 2004). Interestingly, increased neuronal discharges evoked by local application of bicuculline were associated with increased $\left[\mathrm{Ca}^{2+}\right]_{1}$ activity in individual astrocytes and with strongly coordinated $\left[\mathrm{Ca}^{2+}\right]_{1}$ signals among neighboring astrocytes, thus demonstrating the occurrence of dynamic neuron-glia communication in the intact brain (Hirase and others 2004). More recently, several in vivo studies have taken advantage of the functional organization of the barrel cortex. These experiments were undertaken using two properties of in situ astrocytes: their ability to be identified by staining with sulforhodamine 101 and their specific loading with certain membranepermeant calcium sensitive dyes (Nimmerjahn and others 2004). By combining these two types of loading, two-photon imaging allowed specific recording of $\left[\mathrm{Ca}^{2+}\right]_{i}$ increases in astrocytes following whisker stimulation triggered by air puffs (Wang and others 2006). This approach demonstrated that $\left[\mathrm{Ca}^{2+}\right]_{1}$ increases are a function of the frequency of stimulation and occur several seconds ( $3 \mathrm{sec}$ delay; peak at $6 \mathrm{sec}$ ) after whisker responses (Fig. 3). In addition, these astrocytic responses are inhibited by metabotropic glutamate receptor antagonists but are not affected by AMPA and NMDA receptor antagonists. These observations suggest that in vivo astrocytes are activated by glutamate input independently from the postsynaptic response. However, their long latency excludes that astrocytes could play a role in modulating microcirculation in response to neuronal activity as proposed from recent studies performed in brain slices (Mulligan and MacVicar 2004; Zonta and others 2003). Indeed, in vivo imaging of intrinsic optical signals and two-photon imaging of local blood flow in layer II through IV 
of the barrel cortex indicate that an increase of blood flow is recorded within $<1 \mathrm{sec}$ after the stimulation of multiple vibrissae (Kleinfeld and others 1998). However, this role of astrocytes in hyperemia could be challenged based on the recent identification of a subset of astrocytes $(5 \%)$ that, in the somatosensory cortex, exhibit short-latency $(\approx 0.5$ sec) $\mathrm{Ca}^{2+}$ responses to sensory stimulation (Winship and others 2007). Altogether, these studies establish that in the barrel cortex sensory stimuli evoke astrocytic $\left[\mathrm{Ca}^{2+}\right]_{1}$ responses in vivo with various latencies that could account for neuroglial and gliovascular interaction.

The mapping of astrocytic responses within the barrel cortex and their temporal relation with neuronal responses was recently achieved by performing calcium imaging in acute slices of the barrel cortex (Schipke and others 2008). In this study, $\left[\mathrm{Ca}^{2+}\right]_{1}$ responses in neurons and astrocytes were recorded after Fluo-4 loading: astrocytes were distinguished from neurons by loading with sulforhodamine 101 (as in the in vivo studies mentioned above). Direct electrical stimulation in layer IV evoked neuronal and astrocytic $\left[\mathrm{Ca}^{2+}\right]_{1}$ responses that could be discriminated by their spatial distribution and their kinetics (Fig. 4). Responding neurons were found to be located within the stimulated barrel in layer IV, but also beyond barrel column borders in layers II/III and V. One second later, a second cell population, identified as astrocytes and restricted to the stimulated barrel (Fig. 4), responded with a slower rise in $\left[\mathrm{Ca}^{2+}\right]_{1} \cdot\left[\mathrm{Ca}^{2+}\right]_{1}$ transients in astrocytes peaked with a delay of 1-2 seconds after stimulation. These observations demonstrate that astrocytes selectively respond to neuronal activity emerging from layer IV and that their $\left[\mathrm{Ca}^{2+}\right]_{\text {, }}$ response is restricted to a single "innervation barrel" of layer IV (Schipke and others 2008).

\section{6/ Metabolic interactions between neurons and astrocytes}

An adequate and timely production of ATP by brain cells is necessary to support the major energetic costs inherent to the rapid information processing that occurs within the somatosensory cortex during whisker-mediated exploration. As somatosensory whiskerrelated processing is organized into stereotypical spatio-temporal patterns, one can design stimulation protocols with the aim of evoking differential energy use across 
barrels. The barrel cortex therefore provides an attractive model for investigating the molecular mechanisms that ensure a tight coupling between synaptic activity and energy expenditure. This is of critical importance since neuronal processing power is limited by the energy supply available (Attwell and Gibb 2005) and the finite energy budgets of animals could account for many of the morphological features of sensory systems and also for their evolution (Niven and Laughlin 2008).

The brain has a high rate of aerobic metabolism and a respiratory quotient of nearly 1 , indicating that oxidative metabolism of glucose is the major pathway for energy production. This implies that spatially localized and temporally restricted increases over basal glucose use should occur in register with neuronal activity in brain areas subserving specific modalities. The 2-deoxyglucose technique developed by Sokoloff and colleagues (1977) provided experimental validation of this principle (Sokoloff and others 1977). As an example, a tight spatial correlation between blood flow increases and glucose utilization restricted to the activated barrel has been established (Fig. 5).

A continuous supply of glucose and oxygen is mandatory to sustain neuronal activity. An important issue to be addressed is the nature of the neuronal processes that require energy during functional activation. The prevailing hypothesis is that most of the energy is used to restore ionic gradients and resting membrane potentials that are modified during the excitation phase (Sokoloff 1992). This restoration is mainly provided by the $\mathrm{Na}^{+} / \mathrm{K}^{+}$ATPase that consumes ATP to re-establish $\mathrm{Na}^{+}$and $\mathrm{K}^{+}$gradients. Elegant theoretical calculations of the cost of synaptic transmission have shown that $87 \%$ of total energy consumed reflects the activity of glutamate-mediated neurotransmission while the rest reflects the energy requirements of resting potential maintenance in neurons and glia (Attwell and Laughlin 2001). With the advent of in vivo Magnetic Resonance Spectroscopy, it has been possible to show that energy consumption is indeed mainly devoted to glutamatergic neurotransmission (Escartin and others 2006; Rothman and others 2003). Although these studies demonstrated a quantitative relationship between cortical energy expenditure and glutamate-mediated neurotransmission, they did not relate glucose consumption to specific processes directly. In other words, it is not known 
which signaling pathway enables tight adjustment of glucose use to the increasing needs of the glutamatergic synapses. In the barrel cortex, it was then realized that astrocytes, via their privileged anatomical relationships among brain capillaries (Kacem and others 1998) and synapses. Figure 6 gives a few examples of astrocytic processes in the vicinity of synapses, excitatory as well as inhibitory (Fig. 6 A and B, respectively), in the barrel cortex of the mouse This spatially relationship places astrocytes in an ideally position to sense synaptic activity and to couple it with uptake and metabolism of energy substrates originating from the circulation.

How can astrocytes sense synaptic activity? One likely possibility is via detection of changes in the concentration of particular ions or neurotransmitters. Neuronal excitation generates action potentials produced by a depolarization-induced rapid inward $\mathrm{Na}^{+}$ current and $\mathrm{K}^{+}$efflux. Numerous experiments have been conducted in cultured astrocytes to determine the effects of elevated extracellular $\mathrm{K}+$ and/or intracellular $\mathrm{Na}+$ on glucose metabolism. Astrocytic glucose metabolism is stimulated by increased intracellular $\mathrm{Na}^{+}$(Escartin and others 2006) but insensitive to high extracellular $\mathrm{K}^{+}$. As for detection of neurotransmitters, experiments performed on cultures have shown that glutamate stimulates 2-DG uptake and phosphorylation by astrocytes in a concentrationdependent manner (Pellerin and Magistretti 1994; Takahashi and others 1995). The effect of glutamate is mediated by $\mathrm{Na}^{+}$-dependent glutamate uptake, which is performed in astrocytes by two glutamate transporters, GLAST and GLT-1, independently of glutamate receptors. These transporters were demonstrated to be inserted in the membrane of astrocytic processes close to excitatory synapses (see Fig. 6C for an example). Glutamate uptake is powered by the cotransport of three $\mathrm{Na}^{+}$ions and one $\mathrm{H}^{+}$ while one $\mathrm{K}^{+}$is counter-transported. Experiments have confirmed that it is indeed the intracellular concentration of $\mathrm{Na}^{+}$and not glutamate itself that activates the $\mathrm{Na}^{+} / \mathrm{K}^{+}$ ATPase pump, decreases ATP levels and activates glycolysis in astrocytes (Chatton and others 2000). Until recently, no information was available on the putative role of these transporters in vivo. Interestingly, a specific enrichment of GLT-1/GLAST expression observed in the barrel hollows (Fig. 7) during development provides a useful model to study the role of these glial glutamate transporters in the regulation of brain 
energy metabolism in vivo. Indeed, it was shown that the metabolic response, as observed by 2-DG autoradiography in living animals of different ages, was altered in knockout mice for each of these glutamate transporters (Voutsinos-Porche and others 2003a; Voutsinos-Porche and others 2003b). The metabolic response to whisker stimulation was halved in P9-P10 GLT-1 and GLAST -/- mice. However, the role of GLAST is transient since the metabolic response is restored in P11-P12 GLAST -/mice and unchanged in adult GLAST $-/-$ mice, while deletion of GLT-1 continuously impaired the functional response until adulthood (Fig. 2 GB).

These results are in agreement with the observation that the development of the total glutamate uptake activity in the cortex parallels that of GLT-1, so that GLT-1 accounts for $\sim 90 \%$ of the total transport activity in adult (Ullensvang and others 1997). Therefore, a correlation exists between the capacity of glutamate uptake and the uptake of glucose during whisker activation for both glutamate transporters during development. These data provided evidence that astroglial glutamate transporters are key components of a metabolic signaling pathway that leads to the transfer of energy substrates to activated glutamatergic neurons in the mouse somatosensory cortex. A tight dialogue (initiated by the uptake of glutamate) between neurons and astrocytes is therefore of critical importance to support the rapid processing of information (mediated by glutamate) that occurs within the somatosensory cortex.

\section{7/ Integrated role of astrocytes in barrel plasticity}

Due to its morphological characteristics, the whisker-to-barrel system has been extensively used to investigate brain plasticity (Petersen 2007). These studies can be subdivided roughly into those that identified the factors underlying the development of the pathway and those that tested the factors that maintain the integrity of the ascending pathway during adulthood. As in other sensory pathways, most of the studies on plasticity in the whisker-to-barrel pathways use sensory deprivation as an experimental protocol. Since the initial study of Van der Loos and Woolsey (1973), whisker follicles have been lesioned or de-afferented, whiskers have been clipped or plucked in laboratories around the world. These different peripheral manipulations lead to a 
decrease in sensory input into the pathway and affect the morphological and/or physiological organization of representations. Sensory deprivation studies have led to the concept of a "critical period" based on the observations that the structural pattern of the whisker representations can be altered if applied before the fourth postnatal day. The exact timing of the critical period is different for the stations within the ascending pathway: earliest for the representation in the brainstem; latest, in the cerebral cortex (Durham and Woolsey 1984). Beyond the critical period, sensory deprivation has been shown to alter response properties of neurons within the central representations, as well as synaptic connectivity (Frostig 2006). As a counterpart of sensory deprivation, two other protocols have been used to induce plasticity by increasing sensory input into the pathway: exposing animals to enriched environments (Polley and others 1999) and selective whisker stimulation (Melzer and others 1985).

Astrocytes have been identified to contribute to plasticity in the barrel cortex induced by altered sensory input. A first set of observations is related to the high level of expression of the astrocyte-derived extracellular matrix glycoprotein tenascin- $\mathrm{C}$ during the period of barrel formation (Crossin and others 1989; Jhaveri and others 1991; Laywell and Steindler 1991; Mitrovic and others 1994; Mitrovic and others 1996). This glycoprotein is initially uniformly present in layer IV, but its expression is reduced in the center of the barrel where thalamo-cortical axons segregate (Mitrovic and others 1994). Blockade of NMDA-receptors during barrel formation prevents the down-regulation of tenascin-C within the developing barrel (Mitrovic and others 1996), indicating that the level of tenascin- $C$ is regulated by neuronal activity and could therefore play a role in sensoryactivity dependent plasticity during barrel development. Interestingly, in tenascin-C deficient mice the cytoarchitectonic differentiation into barrels is normal (Steindler and others 1995) although the whisker-evoked responses are abnormal (Irintchev and others 2005). Removing all mystacial whisker follicles in the adult wild-type mouse except those of row $\mathrm{C}$ leads to an expansion of the functional representation of the spared whiskers. In the tenascin-C deficient mouse this functional expansion is reduced (CybulskaKlosowicz and others 2004), which is not due to a difference in (re)innervation pattern of the remaining follicles between the mutant and wild-type mice. Alternatively, it has been 
proposed that this could be the consequence of the altered ratio between excitatory/inhibitory neurons in the mutant mouse (Irintchev and others 2005).

Another remarkable observation indicating an important role of astrocytes in barrel formation and plasticity is the high level of expression of astrocytic glutamate transporters within the developing barrel. GLAST or GLT-1 deficient mice develop barrels normally although the level of whisker-stimulation induced deoxyglucose uptake is transiently reduced in these mice during postnatal development (see chapter 6). Recently, in GLT-1 deficient mouse the level of extra-cellular glutamate was found to be increased during the same period in which whisker-evoked metabolic activity is reduced (Takasaki and others 2008). These authors further demonstrated a role of glutamate transporters in regulating the extent of lesion-induced plasticity in the barrel cortex. In wild-type mice, postnatal lesions of the follicles of row $C$ perturbs the formation of the corresponding barrels and induces the expansion of the barrels of the neighboring rows (Van der Loos and Woolsey, 1973). However, Takasaki et al. (2008) demonstrated that in both GLT-1 and GLAST deficient mice the expansion of the neighboring barrels is reduced while a representation of the row $C$ follicles is present in mice two weeks after the peripheral intervention. Taken together these observations identify a role for astrocytes in controlling morphological plasticity during barrel development by regulating the level of glutamate in the extra-cellular space. This function is, obviously, not only of importance during cortical development. Indeed, the question was raised how the level of GLT-1 and GLAST is regulated by sensory activity within the whisker-to-barrel pathway. Using the Lausanne-whisker stimulator, Genoud et al. (2006) showed that the protein-levels of both glutamate transporters are up-regulated after 24 hours of continuous sensory stimulation. This biochemical observation was paralleled with the ultrastructural observation of an increased astrocytic coverage of excitatory synapses within the stimulated barrels. Two other observations are useful in the interpretation of these results: this stimulation paradigm leads to an increase in the ratio between inhibitory and excitatory synapses within the stimulated barrel (Knott and others 2002) and, on a functional level, leads to habituation of the cortical neurons to sensory input from the stimulated whisker (Quairiaux and others 2007; Welker and others 1992). It is 
therefore proposed that the levels of GLT-1 and GLAST expression in astrocytes contribute to a homeostatic mechanism through which the overall level of neuronal activity within the barrel is kept within a physiological range. This unique example underscores the partnership between neurons and astrocytes in sensory processing.

\section{8/ Conclusions and perspectives}

As indicated throughout this review, there are several lines of evidence for dynamic interaction between neurons and astrocytes in the barrel cortex. The occurrence of this dialog between two major cell types of the nervous system is likely favored by the tight contact of astrocytic processes with excitatory and inhibitory synaptic sites observed at the ultrastructural level. Indeed, released neurotransmitters activate receptors and are taken up by transporters in astrocytes, evoking $\mathrm{Ca}^{2+}$ and $\mathrm{Na}^{+}$increases that might propagate as intra- and intercellular waves (Bernardinelli and others 2004; Scemes and Giaume 2006) (Fig. 8). Such a mechanism could account for the findings observed in vivo that whisker stimulation is able to trigger calcium responses in astrocytes of the somatosensory cortex (Wang et al., 2006). Interestingly, brain slice experiments indicate that calcium increases in astrocytes are even restricted to the barrel unit whereas neuronal activation secondarily diffuses to other barrel columns at the level of layer II/III. In addition, these neuroglial interactions are plastic since prolonged whisker stimulation induced notable changes in both the astrocytic coverage of synapses and the expression of glutamate transporters (Fig. 8). Previously, in the hypothalamus such changes have been reported to have a marked consequence on neuronal activity (Theodosis and others 2008). Accordingly, it is tempting to hypothesize that in the barrel cortex also, changes in the properties of glia may also affect neuronal behavior. The study of astrocytes in the barrel cortex has also contributed to establish the emerging notion that these glial cells represent a heterogeneous cell population, as already observed by gene profiling (Barres 2008) and functional studies (Kettenmann and Verkhratsky 2008). Even if the distribution of astrocytes is homogenous within the barrel field, immunolabeling of proteins known to be highly expressed by astrocytes (GLT-1 and GLAST, Cx43 and Cx30, glutamine synthetase) indicated that in layer IV, astrocytes located within or between the barrels do not share the same level of expression. Such 
difference can be either transitory (until P10 for GLT1 and GLAST) or permanent (Cx43 and Cx30). However, so far this heterogeneity has only been investigated at the functional level for connexins (Houades et al., 2008). Finally, when comparing astroglial and neuronal networks, it is noteworthy that both follow similar rules for the direction and strength of spatial organization (Fig. 9). As a general feature, gap junction-mediated coupling in cortical layers I and II/III, and in layer $\mathrm{V}$ to a lesser extent, is oriented transversally (Houades et al., 2006); in contrast this coupling is restricted between two barrels in layer 4 (Houades et al., 2008). Similarly, neuronal connections across barrel columns occur in layer II/III and V while in layer IV neurons do not connect across barrels (Fig. 9). In addition, within each barrel, gap junctional communication is oriented toward its center, where neuronal dendrites also tend to cluster in the rat (although not in the mouse).

As discussed above, the barrel cortex offers many useful properties allowing further progress in the analysis and the understanding of the contribution of astrocytes to dynamic neuroglial interaction. In the near future, these approaches should help to address questions related to basic central functions. Regarding circuit function, one question that needs to be explored is: how sharply tuned to whisker identity or direction are astrocytic responses? In visual cortex, astrocytes are very sharply tuned to sensory stimuli (Schummers and others 2008). Response tuning would give important hints as to the nature of the wiring of astrocytes with respect to neurons. For example, if astrocytic circuitry is functionally dominated by gap junction-mediated coupling within a barrel, one should expect each astrocyte in the barrel to respond non-specifically: all should respond similarly to the principal whisker, and also share directional tuning. Thus, similar responses of neighboring astrocytes to different whiskers would be evidence in favor of the functional importance of cell-to-cell coupling; conversely, changes in whisker selectivity on a cell-to-cell basis, as observed for neurons (Sato and others 2007), would suggest that the key determinant of astrocytic activation is the intimate connection with nearby neurons, rather than network coupling. 
Regarding how the circuit structure is set up, it would be instructive to study the role of neuronal activity in shaping the spatial organization and the differentiation of astrocytes at the single cell as well as at the network level. This could help determine whether a critical period occurs for glial organization, as is the case for neurons. Conversely, the contribution of astrocytes and neuroglial interactions to the maturation of neuronal intrinsic properties and circuitry could also be addressed. To answer these questions it is crucial to identify the mechanisms involved in calcium signaling within and between astrocytes. Indeed, while it is now well admitted that calcium signaling provides a basis for glial excitability (Scemes and Giaume 2006), the existence of, and mechanisms involved in the calcium-dependent release of gliotransmitters are still debated (Agulhon and others 2008; Barres 2008). Here also, the barrel cortex could provide a well-adapted model to address this question in vivo by using transgenic animals in which genes encoding for essential elements involved in the release process (SNARE, P2X receptors, Cxs..) have been invalidated. Finally, the availability of several "barrel-less" models (Inan and Crair 2007) offers the opportunity to dissociate neuronal architecture from function. The use of these transgenic animals to search for changes in astrocytic properties and neuroglial interaction could help to explain the observed changes of neuronal architecture and/or functional responses, and will provide a basic understanding of how neurons and glial cells construct in close collaboration the columnar organization of the cerebral cortex during development.

Acknowledgments: The authors thank S. Ingham for excellent technical help with illustrations and Drs. V. Houades, C. Genoud and G. Knott for having contributed to the illustration of this review.

Financial support was provided by the Human Frontier Science Program (RG0043/2004 to M.M.) and the Spanish Ministry of Education and Science (BFU2006-04791/BFI to M.M., cofunded by the European Regional Development Fund; CONSOLIDER CSD2007-00023). 


\section{References}

Agmon A, Connors BW. 1991. Thalamocortical responses of mouse somatosensory (barrel) cortex in vitro. Neuroscience 41(2-3):365-79.

Agulhon C, Petravicz J, McMullen AB, Sweger EJ, Minton SK, Taves SR and others. 2008. What is the role of astrocyte calcium in neurophysiology? Neuron 59(6):932-46.

Alloway KD. 2008. Information processing streams in rodent barrel cortex: the differential functions of barrel and septal circuits. Cereb Cortex 18(5):979-89.

Arabzadeh E, Panzeri S, Diamond ME. 2006. Deciphering the spike train of a sensory neuron: counts and temporal patterns in the rat whisker pathway. J Neurosci 26(36):9216-26.

Arabzadeh E, Zorzin E, Diamond ME. 2005. Neuronal encoding of texture in the whisker sensory pathway. PLoS Biol 3(1):e17.

Araque A, Parpura V, Sanzgiri RP, Haydon PG. 1999. Tripartite synapses: glia, the unacknowledged partner. Trends Neurosci 22(5):208-15.

Armstrong-James M, Fox K. 1987. Spatiotemporal convergence and divergence in the rat S1 "barrel" cortex. J Comp Neurol 263(2):265-81.

Attwell D, Gibb A. 2005. Neuroenergetics and the kinetic design of excitatory synapses. Nat Rev Neurosci 6(11):841-9.

Attwell D, Laughlin SB. 2001. An energy budget for signaling in the grey matter of the brain. J Cereb Blood Flow Metab 21(10):1133-45.

Barres BA. 2008. The mystery and magic of glia: a perspective on their roles in health and disease. Neuron 60(3):430-40.

Bernardinelli Y, Magistretti PJ, Chatton JY. 2004. Astrocytes generate Na+-mediated metabolic waves. Proc Natl Acad Sci U S A 101(41):14937-42.

Brecht M. 2007. Barrel cortex and whisker-mediated behaviors. Curr Opin Neurobiol 17(4):408-16.

Bruno RM, Sakmann B. 2006. Cortex is driven by weak but synchronously active thalamocortical synapses. Science 312(5780):1622-7.

Bureau I, von Saint Paul F, Svoboda K. 2006. Interdigitated paralemniscal and lemniscal pathways in the mouse barrel cortex. PLoS Biol 4(12):e382.

C KHaS. 2005. Receptors for neurotransmitters and hormones. Neuroglia, editor. Oxford: Oxford University Press.

Carvell GE, Simons DJ. 1990. Biometric analyses of vibrissal tactile discrimination in the rat. J Neurosci 10(8):2638-48.

Cases O, Seif I, Grimsby J, Gaspar P, Chen K, Pournin S and others. 1995. Aggressive behavior and altered amounts of brain serotonin and norepinephrine in mice lacking MAOA. Science 268(5218):1763-6.

Chatton JY, Marquet P, Magistretti PJ. 2000. A quantitative analysis of L-glutamateregulated $\mathrm{Na}+$ dynamics in mouse cortical astrocytes: implications for cellular bioenergetics. Eur J Neurosci 12(11):3843-53.

Cooper NG, Steindler DA. 1986. Monoclonal antibody to glial fibrillary acidic protein reveals a parcellation of individual barrels in the early postnatal mouse somatosensory cortex. Brain Res 380(2):341-8. 
Cornell-Bell AH, Finkbeiner SM, Cooper MS, Smith SJ. 1990. Glutamate induces calcium waves in cultured astrocytes: long-range glial signaling. Science 247(4941):470-3.

Cotrina ML, Nedergaard M. 2002. Astrocytes in the aging brain. J Neurosci Res 67(1):110.

Crossin KL, Hoffman S, Tan SS, Edelman GM. 1989. Cytotactin and its proteoglycan ligand mark structural and functional boundaries in somatosensory cortex of the early postnatal mouse. Dev Biol 136(2):381-92.

Cruikshank SJ, Lewis TJ, Connors BW. 2007. Synaptic basis for intense thalamocortical activation of feedforward inhibitory cells in neocortex. Nat Neurosci 10(4):462-8.

Cybulska-Klosowicz A, Zakrzewska R, Pyza E, Kossut M, Schachner M. 2004. Reduced plasticity of cortical whisker representation in adult tenascin-C-deficient mice after vibrissectomy. Eur J Neurosci 20(6):1538-44.

Dani JW, Chernjavsky A, Smith SJ. 1992. Neuronal activity triggers calcium waves in hippocampal astrocyte networks. Neuron 8(3):429-40.

Daw MI, Scott HL, Isaac JT. 2007. Developmental synaptic plasticity at the thalamocortical input to barrel cortex: mechanisms and roles. Mol Cell Neurosci 34(4):493-502.

Deitmer JW, McCarthy KD, Scemes E, Giaume C. 2006. Information processing and transmission in glia: calcium signaling and transmitter release. Glia 54(7):639-41.

Diamond ME, Armstrong-James M, Budway MJ, Ebner FF. 1992. Somatic sensory responses in the rostral sector of the posterior group (POm) and in the ventral posterior medial nucleus (VPM) of the rat thalamus: dependence on the barrel field cortex. J Comp Neurol 319(1):66-84.

Diamond ME, von Heimendahl M, Knutsen PM, Kleinfeld D, Ahissar E. 2008. 'Where' and 'what' in the whisker sensorimotor system. Nat Rev Neurosci 9(8):601-12.

Durham D, Woolsey TA. 1984. Effects of neonatal whisker lesions on mouse central trigeminal pathways. J Comp Neurol 223(3):424-47.

Escartin C, Valette J, Lebon V, Bonvento G. 2006. Neuron-astrocyte interactions in the regulation of brain energy metabolism: a focus on NMR spectroscopy. J Neurochem 99(2):393-401.

Feldmeyer D, Egger V, Lubke J, Sakmann B. 1999. Reliable synaptic connections between pairs of excitatory layer 4 neurones within a single 'barrel' of developing rat somatosensory cortex. J Physiol $521 \mathrm{Pt}$ 1:169-90.

Feldmeyer D, Roth A, Sakmann B. 2005. Monosynaptic connections between pairs of spiny stellate cells in layer 4 and pyramidal cells in layer 5A indicate that lemniscal and paralemniscal afferent pathways converge in the infragranular somatosensory cortex. J Neurosci 25(13):3423-31.

Fiacco TA, McCarthy KD. 2006. Astrocyte calcium elevations: properties, propagation, and effects on brain signaling. Glia 54(7):676-90.

Frostig RD. 2006. Functional organization and plasticity in the adult rat barrel cortex: moving out-of-the-box. Curr Opin Neurobiol 16(4):445-50.

Gabernet L, Jadhav SP, Feldman DE, Carandini M, Scanziani M. 2005. Somatosensory integration controlled by dynamic thalamocortical feed-forward inhibition. Neuron 48(2):315-27.

Galarreta M, Hestrin S. 1999. A network of fast-spiking cells in the neocortex connected by electrical synapses. Nature 402(6757):72-5. 
Genoud C, Quairiaux C, Steiner P, Hirling H, Welker E, Knott GW. 2006. Plasticity of astrocytic coverage and glutamate transporter expression in adult mouse cortex. PLoS Biol 4(11):e343.

Giaume C, Kirchhoff F, Matute C, Reichenbach A, Verkhratsky A. 2007. Glia: the fulcrum of brain diseases. Cell Death Differ 14(7):1324-35.

Giaume C, McCarthy KD. 1996. Control of gap-junctional communication in astrocytic networks. Trends Neurosci 19(8):319-25.

Gibson JR, Beierlein M, Connors BW. 1999. Two networks of electrically coupled inhibitory neurons in neocortex. Nature 402(6757):75-9.

Gil Z, Amitai Y. 1996. Properties of convergent thalamocortical and intracortical synaptic potentials in single neurons of neocortex. J Neurosci 16(20):6567-78.

Gil Z, Connors BW, Amitai Y. 1999. Efficacy of thalamocortical and intracortical synaptic connections: quanta, innervation, and reliability. Neuron 23(2):385-97.

Guic-Robles E, Valdivieso C, Guajardo G. 1989. Rats can learn a roughness discrimination using only their vibrissal system. Behav Brain Res 31(3):285-9.

Halassa MM, Fellin T, Haydon PG. 2007. The tripartite synapse: roles for gliotransmission in health and disease. Trends Mol Med 13(2):54-63.

Haydon PG, Carmignoto G. 2006. Astrocyte control of synaptic transmission and neurovascular coupling. Physiol Rev 86(3):1009-31.

Hirase H, Qian L, Bartho P, Buzsaki G. 2004. Calcium dynamics of cortical astrocytic networks in vivo. PLoS Biol 2(4):E96.

Houades V, Koulakoff A, Ezan P, Seif I, Giaume C. 2008. Gap junction-mediated astrocytic networks in the mouse barrel cortex. J Neurosci 28(20):5207-17.

Houades V, Rouach N, Ezan P, Kirchhoff F, Koulakoff A, Giaume C. 2006. Shapes of astrocyte networks in the juvenile brain. Neuron Glia Biol 2(1):3-14.

Hutson KA, Masterton RB. 1986. The sensory contribution of a single vibrissa's cortical barrel. J Neurophysiol 56(4):1196-223.

Inan M, Crair MC. 2007. Development of cortical maps: perspectives from the barrel cortex. Neuroscientist 13(1):49-61.

Irintchev A, Rollenhagen A, Troncoso E, Kiss JZ, Schachner M. 2005. Structural and functional aberrations in the cerebral cortex of tenascin- $C$ deficient mice. Cereb Cortex 15(7):950-62.

Jhaveri S, Erzurumlu RS, Crossin K. 1991. Barrel construction in rodent neocortex: role of thalamic afferents versus extracellular matrix molecules. Proc Natl Acad Sci U $S$ A 88(10):4489-93.

Kacem K, Lacombe P, Seylaz J, Bonvento G. 1998. Structural organization of the perivascular astrocyte endfeet and their relationship with the endothelial glucose transporter: a confocal microscopy study. Glia 23(1):1-10.

Kettenmann H, Verkhratsky A. 2008. Neuroglia: the 150 years after. Trends Neurosci 31(12):653-9.

Kim U, Ebner FF. 1999. Barrels and septa: separate circuits in rat barrels field cortex. J Comp Neurol 408(4):489-505.

Kleinfeld D, Mitra PP, Helmchen F, Denk W. 1998. Fluctuations and stimulus-induced changes in blood flow observed in individual capillaries in layers 2 through 4 of rat neocortex. Proc Natl Acad Sci U S A 95(26):15741-6. 
Knott GW, Quairiaux C, Genoud C, Welker E. 2002. Formation of dendritic spines with GABAergic synapses induced by whisker stimulation in adult mice. Neuron 34(2):265-73.

Knutsen PM, Pietr M, Ahissar E. 2006. Haptic object localization in the vibrissal system: behavior and performance. J Neurosci 26(33):8451-64.

Krupa DJ, Matell MS, Brisben AJ, Oliveira LM, Nicolelis MA. 2001. Behavioral properties of the trigeminal somatosensory system in rats performing whisker-dependent tactile discriminations. J Neurosci 21(15):5752-63.

Land PW, Buffer SA, Jr., Yaskosky JD. 1995. Barreloids in adult rat thalamus: threedimensional architecture and relationship to somatosensory cortical barrels. J Comp Neurol 355(4):573-88.

Laywell ED, Steindler DA. 1991. Boundaries and wounds, glia and glycoconjugates. Cellular and molecular analyses of developmental partitions and adult brain lesions. Ann N Y Acad Sci 633:122-41.

Lee KJ, Woolsey TA. 1975. A proportional relationship between peripheral innervation density and cortical neuron number in the somatosensory system of the mouse. Brain Res 99(2):349-53.

Lichtenstein SH, Carvell GE, Simons DJ. 1990. Responses of rat trigeminal ganglion neurons to movements of vibrissae in different directions. Somatosens Mot Res $7(1): 47-65$.

Liu XB, Jones EG. 2003. Fine structural localization of connexin-36 immunoreactivity in mouse cerebral cortex and thalamus. J Comp Neurol 466(4):457-67.

Manns ID, Sakmann B, Brecht M. 2004. Sub- and suprathreshold receptive field properties of pyramidal neurones in layers $5 \mathrm{~A}$ and $5 \mathrm{~B}$ of rat somatosensory barrel cortex. J Physiol 556(Pt 2):601-22.

Mehta SB, Whitmer D, Figueroa R, Williams BA, Kleinfeld D. 2007. Active spatial perception in the vibrissa scanning sensorimotor system. PLoS Biol 5(2):e15.

Melzer P, Van der Loos H, Dorfl J, Welker E, Robert P, Emery D and others. 1985. A magnetic device to stimulate selected whiskers of freely moving or restrained small rodents: its application in a deoxyglucose study. Brain Res 348(2):229-40.

Mitrovic N, Dorries U, Schachner M. 1994. Expression of the extracellular matrix glycoprotein tenascin in the somatosensory cortex of the mouse during postnatal development: an immunocytochemical and in situ hybridization analysis. J Neurocytol 23(6):364-78.

Mitrovic N, Mohajeri H, Schachner M. 1996. Effects of NMDA receptor blockade in the developing rat somatosensory cortex on the expression of the glia-derived extracellular matrix glycoprotein tenascin-C. Eur J Neurosci 8(9):1793-802.

Mulligan SJ, MacVicar BA. 2004. Calcium transients in astrocyte endfeet cause cerebrovascular constrictions. Nature 431(7005):195-9.

Nagy JI, Rash JE. 2000. Connexins and gap junctions of astrocytes and oligodendrocytes in the CNS. Brain Res Brain Res Rev 32(1):29-44.

Nimmerjahn A, Kirchhoff F, Kerr JN, Helmchen F. 2004. Sulforhodamine 101 as a specific marker of astroglia in the neocortex in vivo. Nat Methods 1(1):31-7.

Niven JE, Laughlin SB. 2008. Energy limitation as a selective pressure on the evolution of sensory systems. J Exp Biol 211(Pt 11):1792-804. 
Pellerin L, Magistretti PJ. 1994. Glutamate uptake into astrocytes stimulates aerobic glycolysis: a mechanism coupling neuronal activity to glucose utilization. Proc. Natl. Acad. Sci. U.S.A. 91(22):10625-10629.

Petersen CC. 2007. The functional organization of the barrel cortex. Neuron 56(2):33955.

Petersen CC, Sakmann B. 2000. The excitatory neuronal network of rat layer 4 barrel cortex. J Neurosci 20(20):7579-86.

Petersen CC, Sakmann B. 2001. Functionally independent columns of rat somatosensory barrel cortex revealed with voltage-sensitive dye imaging. J Neurosci 21(21):8435-46.

Petersen RS, Diamond ME. 2000. Spatial-temporal distribution of whisker-evoked activity in rat somatosensory cortex and the coding of stimulus location. J Neurosci 20(16):6135-43.

Pierret T, Lavallee P, Deschenes M. 2000. Parallel streams for the relay of vibrissal information through thalamic barreloids. J Neurosci 20(19):7455-62.

Polley DB, Chen-Bee CH, Frostig RD. 1999. Two directions of plasticity in the sensorydeprived adult cortex. Neuron 24(3):623-37.

Porter JT, Johnson CK, Agmon A. 2001. Diverse types of interneurons generate thalamus-evoked feedforward inhibition in the mouse barrel cortex. J Neurosci 21(8):2699-710.

Quairiaux C, Armstrong-James M, Welker E. 2007. Modified sensory processing in the barrel cortex of the adult mouse after chronic whisker stimulation. J Neurophysiol 97(3):2130-47.

Rebsam A, Seif I, Gaspar P. 2002. Refinement of thalamocortical arbors and emergence of barrel domains in the primary somatosensory cortex: a study of normal and monoamine oxidase a knock-out mice. J Neurosci 22(19):8541-52.

Rothman DL, Behar KL, Hyder F, Shulman RG. 2003. In vivo NMR studies of the glutamate neurotransmitter flux and neuroenergetics: implications for brain function. Annu Rev Physiol 65:401-27.

Sato TR, Gray NW, Mainen ZF, Svoboda K. 2007. The Functional Microarchitecture of the Mouse Barrel Cortex. PLoS Biol 5(7):e189.

Scemes E, Giaume C. 2006. Astrocyte calcium waves: what they are and what they do. Glia 54(7):716-25.

Schipke CG, Haas B, Kettenmann H. 2008. Astrocytes discriminate and selectively respond to the activity of a subpopulation of neurons within the barrel cortex. Cereb Cortex 18(10):2450-9.

Schubert D, Kotter R, Luhmann HJ, Staiger JF. 2006. Morphology, electrophysiology and functional input connectivity of pyramidal neurons characterizes a genuine layer va in the primary somatosensory cortex. Cereb Cortex 16(2):223-36.

Schubert D, Kotter R, Zilles K, Luhmann HJ, Staiger JF. 2003. Cell type-specific circuits of cortical layer IV spiny neurons. J Neurosci 23(7):2961-70.

Schummers J, Yu H, Sur M. 2008. Tuned responses of astrocytes and their influence on hemodynamic signals in the visual cortex. Science 320(5883):1638-43.

Shepherd GM, Svoboda K. 2005. Laminar and columnar organization of ascending excitatory projections to layer $2 / 3$ pyramidal neurons in rat barrel cortex. J Neurosci 25(24):5670-9. 
Simons DJ. 1978. Response properties of vibrissa units in rat SI somatosensory neocortex. J Neurophysiol 41(3):798-820.

Simons DJ, Carvell GE. 1989. Thalamocortical response transformation in the rat vibrissa/barrel system. J Neurophysiol 61(2):311-30.

Sokoloff L. 1992. The brain as a chemical machine. Prog Brain Res 94:19-33.

Sokoloff L, Reivich M, Kennedy C, Des Rosiers MH, Patlak CS, Pettigrew KD and others. 1977. The [14C]deoxyglucose method for the measurement of local cerebral glucose utilization: theory, procedure, and normal values in the conscious and anesthetized albino rat. J Neurochem 28(5):897-916.

Steindler DA, Settles D, Erickson HP, Laywell ED, Yoshiki A, Faissner A and others. 1995. Tenascin knockout mice: barrels, boundary molecules, and glial scars. J Neurosci 15(3 Pt 1):1971-83.

Sun QQ, Huguenard JR, Prince DA. 2006. Barrel cortex microcircuits: thalamocortical feedforward inhibition in spiny stellate cells is mediated by a small number of fastspiking interneurons. J Neurosci 26(4):1219-30.

Swadlow HA, Gusev AG. 2000. The influence of single VB thalamocortical impulses on barrel columns of rabbit somatosensory cortex. J Neurophysiol 83(5):2802-13.

Takahashi S, Driscoll BF, Law MJ, Sokoloff L. 1995. Role of sodium and potassium ions in regulation of glucose metabolism in cultured astroglia. Proc. Natl. Acad. Sci. U.S.A. 92(10):4616-4620.

Takasaki C, Okada R, Mitani A, Fukaya M, Yamasaki M, Fujihara Y and others. 2008. Glutamate transporters regulate lesion-induced plasticity in the developing somatosensory cortex. J Neurosci 28(19):4995-5006.

Theodosis DT, Poulain DA, Oliet SH. 2008. Activity-dependent structural and functional plasticity of astrocyte-neuron interactions. Physiol Rev 88(3):983-1008.

Ullensvang K, Lehre KP, Storm-mathisen J, Danbolt NC. 1997. Differential developmental expression of the two rat brain glutamate transporter proteins GLAST and GLT. Eur J Neurosci 9(8):1646-1655.

Urbain N, Deschenes M. 2007. A new thalamic pathway of vibrissal information modulated by the motor cortex. J Neurosci 27(45):12407-12.

Van der Loos H, Woolsey TA. 1973. Somatosensory cortex: structural alterations following early injury to sense organs. Science 179(71):395-8.

Verkhratsky A, Toescu EC. 2006. Neuronal-glial networks as substrate for CNS integration. J Cell Mol Med 10(4):826-36.

Volterra A, Meldolesi J. 2005. Astrocytes, from brain glue to communication elements: the revolution continues. Nat Rev Neurosci 6(8):626-40.

Volterra A MP, Haydon PG. 2002. The tripartite synapse. Oxford: Oxford University Press. $271 \mathrm{p}$.

Voutsinos-Porche B, Bonvento G, Tanaka K, Steiner P, Welker E, Chatton JY and others. 2003a. Glial Glutamate Transporters Mediate a Functional Metabolic Crosstalk between Neurons and Astrocytes in the Mouse Developing Cortex. Neuron 37(2):275-86.

Voutsinos-Porche B, Knott G, Tanaka K, Quairiaux C, Welker E, Bonvento G. 2003b. Glial glutamate transporters and maturation of the mouse somatosensory cortex. Cereb Cortex 13(10):1110-21.

Wang X, Lou N, Xu Q, Tian GF, Peng WG, Han X and others. 2006. Astrocytic Ca2+ signaling evoked by sensory stimulation in vivo. Nat Neurosci 9(6):816-23. 
Welker E, Rao SB, Dorfl J, Melzer P, van der Loos H. 1992. Plasticity in the barrel cortex of the adult mouse: effects of chronic stimulation upon deoxyglucose uptake in the behaving animal. J Neurosci 12(1):153-70.

Welker E, Van der Loos H. 1986. Quantitative correlation between barrel-field size and the sensory innervation of the whiskerpad: a comparative study in six strains of mice bred for different patterns of mystacial vibrissae. J Neurosci 6(11):3355-73.

Wilent WB, Contreras D. 2005. Dynamics of excitation and inhibition underlying stimulus selectivity in rat somatosensory cortex. Nat Neurosci 8(10):1364-70.

Winship IR, Plaa N, Murphy TH. 2007. Rapid astrocyte calcium signals correlate with neuronal activity and onset of the hemodynamic response in vivo. J Neurosci 27(23):6268-72.

Woolsey TA, Van der Loos H. 1970. The structural organization of layer IV in the somatosensory region $(\mathrm{SI})$ of mouse cerebral cortex. The description of a cortical field composed of discrete cytoarchitectonic units. Brain Res 17(2):205-42.

Yu C, Derdikman D, Haidarliu S, Ahissar E. 2006. Parallel thalamic pathways for whisking and touch signals in the rat. PLoS Biol 4(5):e124.

Zonta M, Angulo MC, Gobbo S, Rosengarten B, Hossmann KA, Pozzan T and others. 2003. Neuron-to-astrocyte signaling is central to the dynamic control of brain microcirculation. Nat Neurosci 6(1):43-50. 


\section{Legends:}

Figure 1: Flow of lemniscal information within neurons in a barrel column. Arrows depict the main information streams: lemniscal input from the VPM thalamic nucleus to layer IV, feedforward input from layer IV to supragranular layers, interlaminar connections between supragranular to infragranular layers, and cross-columnar interactions within supragranular and infragranular layers. The relatively strong recurrent connectivity within a barrel is shown as a spreading red cloud. Corticocortical outputs are mainly from the supragranular layers. Extracortical outputs are depicted in different colors: green for subcortical outputs from layer $\mathrm{Va}$, pink for subcortical outputs from $\mathrm{Vb}$, gray for corticothalamic outputs from layer VI.

Figure 2: A: Example of dye coupling observed after the injection of biocytin in an astrocytes, identifed by its morphology and electrophysiological properties, located within a barrel of the mouse somatosensory cortex. The green arrows, perpendicular and parallel to the surface of the cortex, indicate the limits of the coupling area after 20 minutes of injection. Note that the intercellular diffusion of biocytin is restricted in the transversal direction. Scale bar: $300 \mu \mathrm{m}$. B: Drawing of the distribution of astrocytes in three adjacent barrels. The green arrows indicate the magnitude of gap junctionmediated communication in the parallel and perpendicular directions. The intensity of blue color of astrocytes indicates the level of $\mathrm{Cx} 43$ and $\mathrm{Cx} 30$ expression. Note that the distribution of astrocytes in the barrel field is considered to be homogenous (see paragraph 3). (see also Houades et al., 2008)

Figure 3: In vivo whiskers stimulation evokes calcium responses in astrocytes from the barrel cortex. A: Diagram of the experimental set-up. B: Example of a dual loading of the barrel cortex with sulforhodamine 101 (left) and fluo-4 AM (right). Note that only astrocytes are loaded with the calcium indicator. Scale bar: $30 \mu \mathrm{m}$. C: Upper trace, local

field potential. Lower trace, $\left[\mathrm{Ca}^{2+}\right]_{i}$ activity monitored in astrocyte number 2 during the whisker stimulation(WS). Lower images illustrate the calcium activity in several 
astrocytes before and during the wisker stimulation. (Modified from Wang and others 2006).

Figure 4: Astrocytes selectively respond to stimulation into a barrel column. A: Focal

electrical stimulation of in layer IV leads to an increase in $\left[\mathrm{Ca}^{2+}\right]_{i}$ in neurons along the barrel and horizontally across barrel structures in layer II/III. In contrast, the delayed responses monitored in astrocytes are restricted to the barrel itself in layer IV. B: Overlay of the regions indicated in A. The neuronal signal during stimulation (blue) does not overlap with the astrocytic calxium signal that appears 1 second latter (violet). Note that calcium responses in astrocytes stop at the barrel border. $\mathbf{C}: \mathrm{A}$ spontaneous $\left[\mathrm{Ca}^{2+}\right]_{\mathrm{i}}$ increase in neurons from layer II/III is not followed by responses in astrocytes. Scale bar : $30 \mu \mathrm{m}$. D: Cartoon of the experimental protocol. (From Schipke and others 2008)

\section{Figure 5: Gilles please complete}

\section{Figure 6: Gilles please complete}

Figure 7: Electron microscopic images to illustrate the spatial proximity of astrocytic processes to synapses in the barrel hollow of the mouse. Synapses are identified by black arrowheads placed in the postsynaptic element. IA and B: Astrocytic processes are highlighted in green to demonstrate their presence close to an excitatory synapse on a dendritic spine(A) and to an inhibitory synapse on a dendritic shaft (B). C: GLASTimmunoreactivity revealed with electron-dense DAB reaction product (white arrowheads) in an astrocytic process surrounding an excitatory synapse on a spine. Scale bar in C correspond to $50 \mathrm{~nm}$ and pertains to all images. The images were kindly provided by Christel Genoud (FMI, Basel, CH) and Graham Knott (EPFL, Lausanne, $\mathrm{CH}$ ).

Figure 8: Bidirectional dialog between neurons and astrocytes. Drawing illustration the dynamic signaling between the two brain cell types. Left part: neurotransmitter release at a synapse stimulates astrocytic receptors that can trigger intra- and intercellular calcium waves, while glutamate up-take through glutamate transporters can generate 
intercellular sodium waves. Right part: astrocytes can release gliotransmitters in a calcium-dependent or independent manner, these compounds activate synaptic and particularly extrasynaptic receptors. The astrocytic processes that contact synapses are also plastic and their coverage of neuronal membranes can be modified in an activitydependent manner.

Figure 9: Astrocytic and neuronal networking between and within barrel columns. Upper drawing: the green arrows indicate the preferential direct intercellular exchanges that occurs between astrocytes of two adjacent barrel columns through gap junctions. Note that the strength of coupling is different depending on the cortical layer as indicated by the thickness of the green arrows. Within a barrel, gap junctional communication is favored towards its center (see Houades and others, 2006; 2008). Lower drawing: across barrel columns, neuronal information flow (red arrows) occurs preferentially within the same layers as astrocytic information flow. Arrow thickness indicates strength of coupling, as above. Within barrels, strong neuronal connectivity parallels astrocytic connectivity. 